\title{
A Learning Method for Stiffness Control of a Drum Robot for Rebounding Double Strokes
}

\author{
Seyed Mojtaba Karbasi \\ RITMO Centre for Interdisciplinary \\ Studies in Rhythm, Time and Motion \\ Department of Informatics \\ University of Oslo \\ Oslo, Norway \\ mojtabak@ifi.uio.no
}

\author{
Rolf Inge Godøy \\ RITMO Centre for Interdisciplinary \\ Studies in Rhythm, Time and Motion \\ Department of Musicology \\ University of Oslo \\ Oslo, Norway \\ r.i.godoy@imv.uio.no \\ Jim Tørresen \\ RITMO Centre for Interdisciplinary \\ Studies in Rhythm, Time and Motion \\ Department of Informatics \\ University of Oslo \\ Oslo, Norway \\ jimtoer@ifi.uio.no
}

\author{
Alexander Refsum Jensenius \\ RITMO Centre for Interdisciplinary \\ Studies in Rhythm, Time and Motion \\ Department of Musicology \\ University of Oslo \\ Oslo, Norway \\ a.r.jensenius@imv.uio.no
}

\begin{abstract}
In robot drumming, performing double stroke rolls is a key ability. Human drummers learn to play double strokes by just trying it several times. For performing it, a model needs to be learned to provide anticipatory commands during drumming. Joint stiffness plays a key role in rebounding double stroke task and should be considered in the model. We have introduced an interactive learning method for a drum robot to learn joint stiffness for rebounding double stroke task. The model is simulated for a $2-\mathrm{DoF}$ robotic arm. The algorithm is simulated with 3 different drum kits to show the robustness of the learning approach. The simulation results also show significant compatibility with human performance results. In addition, the refined learning algorithm adjusts the stroke timing which is important for producing proper rhythms.
\end{abstract}

Keywords-impedance control, drum robot, rebounding double stroke, robot control

\section{INTRODUCTION}

High speed performance can be observed widely in drummers. While the neuromuscular system has a relatively slow dynamical response, skilled drummers can play a drum roll (a sustained sound on a drum) at over $20 \mathrm{~Hz}$. This cannot be achieved without exploiting the natural dynamics of the instrument and anticipatory control strategies. One of the common techniques used by drummers for high speed drum rolls is the rebounding double stroke roll. The double stroke means making two notes within single swing. In a drum roll, which is commonly played by two hands, each hand plays two notes in its turn.

Although the drummer does not necessarily reach the maximum speed by this technique, it embodies the passive dynamic interaction and is straightforward to be measured and analyzed for a motor control or robotic setting. Joint stiffness plays a key role in such a task to be performed in the desired way. In this study, we introduce a learning model for modulating joint stiffness of a 2-DoF robotic arm supposed to perform the rebounding double stroke task.

The double stroke roll starts with one hand striking the drum with the stick, which is allowed to bounce once and produce the double beat. As the stick is retracted, the other hand moves the stick toward the drum membrane for the next double stroke. This altering sequence of double bounces is repeated to generate the drum roll with the desired frequency.

The rebounding effect can be possible with proper grip forces imposed on the pivot of the drumstick. The grip forces can be modeled as the joint stiffness which is a controlled parameter. The stiffness control strategy is explained under the impedance control theory developed by the motor control and robotics researchers during past decades [1, 2]. However, stiffness, or generally impedance, characteristics of the body can not be modulated continuously by the neuromuscular system during very rapid tasks like drum rolls because of the relatively slow dynamics of the neural response [3]. Such a paradoxical paradigm has been described by the motor control theories mostly based on anticipatory strategies [4,5,6]. All these strategies require kind of internal models to be learned via interaction.

In robotic applications, force control approaches have been used for drumming by different researchers. In [7], Hajian et al. have constructed a simple, single-joint robot that emulates the human drumming double stroke technique by modulating passive impedance of McKibben "artificial muscle". They have successfully shown that the robot can perform double stroke drumming by stiffness configuration. However, they did not provide a learning platform and used data collected by a trained human drummer to configure joint stiffness. Berdahl et al. used a similar approach for a haptic drumstick robot [8]. Variable stiffness actuators have been used by Kim et al. for successfully implementing the double stroke task with a single-joint robot [9]. They used an analytical linearized method for adjusting the stiffness of the actuators. However, this method cannot be generalized to a more adaptive and general platform in real applications. In [10], a generative model is used for performing different drum rolls by just a single joint robot. The joint force is configured by a linearized acceleration generated by a griper.

Our study provides a learning strategy for configuring joint stiffness during a double stroke task. We have used a 2- 
DoF robot model for simulating the algorithm. This model has a more similar dynamics to a human hand compared to a 1DoF robot used in previous works.

The main contribution of this paper is introducing a learning method that can adapt to different drum kits with different physical attributes just by playing and listening to the output sound. We are not aware of any learning approach to the problem in similar works. In addition, the algorithm adjusts the timing of the double strokes which is crucial in drumming and music generation applications. Using soundbased feedback data for updating the control parameters is the other advantage of current approach. The interactive learning model can be used for refining joint stiffness in real-time for drum roll application as a part of an anticipatory control scheme. This work is a step in the progress to have an interactive robotic system for drumming which would be able to learn via interaction with the environment.

The rest of the paper is organized as follows: in Section II, we define the control problem by formulating the robot dynamical model, impedance control rule, and drum membrane physical model. In Section III, the learning method is introduced as a solution to stiffness modulation. Section IV explains the simulation results from the dynamical model and the learning algorithm. Finally in Section V, we discuss the conclusion and future work.

\section{METHOD}

\section{A. Robot Model}

The robot model used in this study is shown in Figure 1. The robot consists of two joints controlled by two active actuators using torque control approach. The reason for choosing a 2-DoF arm is that we want to control the stiffness and the position of the drumstick at the same time. The first joint is considered in place of the wrist joint in human arm, which has the largest role in generating desired movements. The second joint is considered in place of the grip which produces the necessary torque to hold the stick while adjusting the stiffness for achieving rebounding behavior.

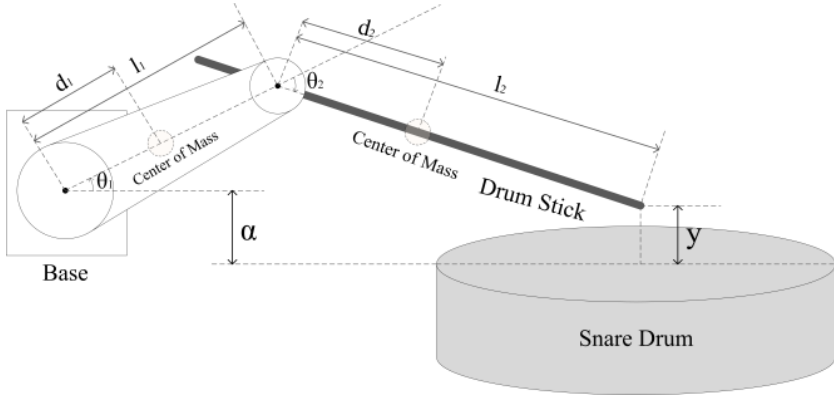

Fig. 1. The 2-DoF drum robot model.

For reducing kinematic complexity, we assume that the position of the first joint is controlled precisely and generates a sinusoidal trajectory continuously with a desired frequency $f$. Thus, the dynamical equation for the second joint can be written as follow:

$$
I \ddot{\theta_{2}}=-m_{2} g d_{2} \cos \left(\theta_{1}+\theta_{2}\right)+\tau_{\text {imp }}+\tau_{\text {ext }}
$$

where $m_{2}$ is the mass of the drumstick, $g$ is the gravitational acceleration, $\tau_{i m p}$ is the control torque generated by the joint impedance, $\tau_{\text {ext }}$ is the external torque causing the bouncing effect by the drum membrane when the stick strikes the drum, and $I$ is the moment of inertia of the stick given by the equation [10]:

$$
I=\frac{m_{2}}{3}\left(3 l_{2}^{2}-3 l_{2} L+L^{2}\right)
$$

where $L$ is the length of the stick.

\section{B. Control Torque}

The control torque in the second joint is modeled as a rotational spring-damper system with adjustable coefficients. In fact, the learning problem for the robot is to find the optimal values for the impedance coefficients of the joint. This approach needs an actuator capable of torque control such as direct drive motors.

The impedance torque is given by the equation:

$$
\tau_{\text {imp }}=-k\left(\theta_{2}-\theta_{d}\right)-b \dot{\theta}_{2}
$$

where, $k$ is the stiffness of the joint, $\theta_{d}$ is the desired angle of the stick which is assumed constant, and $b$ is the damper coefficient. Note that these coefficients are not direction independent as the values vary for each direction of movement of the human arm.

\section{Control Constaints}

As we can observe in human drumming, the amplitude and the frequency of the movements are limited to the physical constraints of the arm and muscles [11]. In the robot model, there are some limitations too; First, the robot dynamics which is introduced by Equation (1) and (2). The robot arm inertia and the gravitational forces are two main factors that define the dynamical behavior of the robot in response to the control and external forces.

Second, the actuators of the robot can generate limited range of torques and therefore, the amplitude of the movements should be adjusted according to the frequency. The second constraint is considered in the simulation model by a saturation function on actuator torques.

\section{Drum Membrane Model}

The drum membrane can be modeled as a massless springdamper system with a large value for stiffness:

$$
F_{d}=-k_{d}\left(\mathrm{y}-y_{d}\right)-b_{d} \dot{y}+n
$$

where $y_{d}$ is the position of the drum membrane, $F_{d}$ is the bouncing force imposed to the tip of the drumstick, $n$ a bandlimited white noise signal, and $k_{d}$ and $b_{d}$ are the impedance coefficients of the drum. This force is imposed at the moment of stroke. The coefficients can be estimated using different measurements, but the learning algorithm should adapt the joint stiffness just by interacting and without measuring the coefficients. We consider 3 different cases for the drum model coefficients to measure the robustness of the algorithm. In addition, we have used a random noise term to consider the uncertainties and nonlinearity of the drum dynamics.

A drummer can learn the skill by trial and error over many instances. Therefore, there is no need for the robot to calculate analytically the joint stiffness according to the stiffness of the drum membrane. In addition, we have used a random noise 
term to consider the uncertainties and nonlinearity of the drum dynamics in the simulation model.

The Force imposed to the tip of the stick generates the external torque at the second joint:

$$
\tau_{\text {ext }}=F_{d} l_{2} \cos \left(\theta_{1}+\theta_{2}\right)
$$

\section{LEARNING ALGORITHM}

The learning algorithm consists of two phases with two separate goals. The goal of the stiffness control is initially to perform the double stroke task. Thus, the error for the first phase can be defined as the difference between the number of strokes in each trial and the desired number of strokes in a certain time frame. This error function can be used for updating the stiffness value in each trial:

$$
k_{n+1}=k_{n} \cdot \alpha^{\varepsilon}
$$

where, $\alpha>1$ is the learning constant, and $\varepsilon$ is the error as described:

$$
\varepsilon=z-v
$$

where, $z$ is the desired number of strokes and $v$ is the actual number of strokes in each trial. In this phase, the stiffness would increase if the error is positive, which means the number of strokes is less than the desired value. This is reasonable because by increasing the stiffness, the frequency will also increase, and it can decrease the error. In addition, the stiffness value is guaranteed to remain positive as it should be.

In the case of the desired performance when we have the double stroke drumming, the error will be zero and the stiffness will not be updated. Thus, we need another error definition to refine performance. The timing of the strokes is another important feature that should be considered during drumming. During double stroke drum rolls with two hands, each hand performs two strokes in a row. Since in each period four notes are played, the time interval between every pair of the double strokes performed by one hand is $1 / 4$ of the time period (and consequently is $1 / 3$ of the time gap between the rebounding stroke and the next stroke by the same hand). This time gap allows the other hand to perform the double stroke. We can define the second error function in order to make the time interval between each pair of double strokes $1 / 4$ of time period. Therefore, the second refining rule for the stiffness can be updated by the following equation:

$$
k_{n+1}=k_{n} \cdot \beta^{\zeta-\epsilon}
$$

where, $\beta>1$ is the learning constant, $\zeta$ is the desired time interval, and $\epsilon$ is the actual time interval between double strokes. The value of $\zeta$ can be defined regarding the frequency as follows:

$$
\zeta=\frac{1}{4 . f}
$$

Each phase runs just once. After the first phase is finished, the training process uses the second phase to maintain the stiffness regarding the time interval of the strokes. The training stops when the second phase ends.
Both errors used for learning algorithm are based on the output sound of the drum robot. However, in the simulation we did not simulate the output sound of the drum membrane to extract the sound features. In the simulation, the number and timing of the strokes are extracted from force output of the drum membrane $\left(F_{d}\right)$.

In the implementation, a microphone can be used to extract the sound features. It means that the robot would be able to listen to its own performance and learn the desired behavior. This two-phase learning process can be performed for each frequency once to establish an internal model for the robot to modulate the joint stiffness during the performance. It can also be used for real-time refinement of the stiffness.

The block diagram of the control and learning scheme is shown in Figure 2.

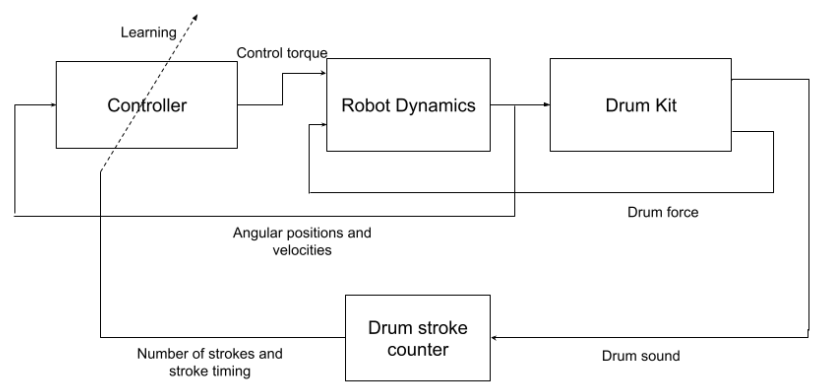

Fig. 2. Control and learning block diagram.

\section{RESULTS}

The proposed model is simulated in MATLAB/Simulink. In each trial, the model was run for 2 seconds. The stiffness value is refined after each trial using Equation (6) and (8). By changing the frequency $f$ from 2.5 to $7 \mathrm{~Hz}$, the optimal stiffness value is learned for each frequency via interaction. We used 3 different drum kit models for testing the robustness of the algorithm. In case 2, the damper coefficient is 10 times larger and in case 3, the stiffness coefficient is 10 times larger than case 1. All the optimal values have been found in less than 20 iterations. The results are significantly compatible with the previous studies in [7]. They showed that in double stroke task, the grip force is proportional to the square of frequency using collected drummer data.

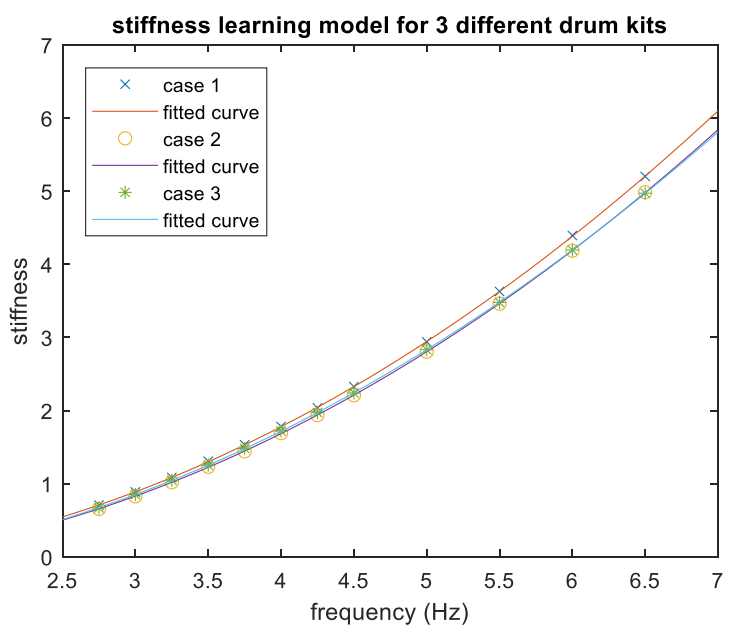

Fig. 3. Stiffness model for different frequencies and drum kits. 
In Figure 3, the trained model is illustrated as the simulated data are fitted to a second-degree polynomial, which supports the results in [7]. In addition, the small difference in the results 3 cases indicates the robustness of the learning algorithm. It means the robot is able to adapt to drum kits with different physical attributes.

In Figure 4, a sample of the signal $y$ (drumstick vertical position) for $f=3 \mathrm{~Hz}$ is shown. In this trial the learning process had only performed the first step and the time difference of the strokes was not adjusted. It can be seen that there are some unwanted jumps between the strokes (shown by red circle) and the timing is not perfect too. After running the second phase of the learning algorithm, both these problems are solved (Figure 5). In Figure 5, the motion is smooth and the time differences between strokes are distributed as desired.

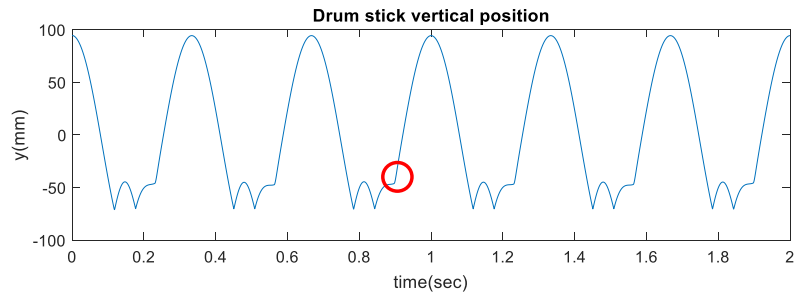

Fig. 4. Simulation:Vertical position of the drumstick in one trial after first learning phase $(\mathrm{f}=3 \mathrm{~Hz})$.

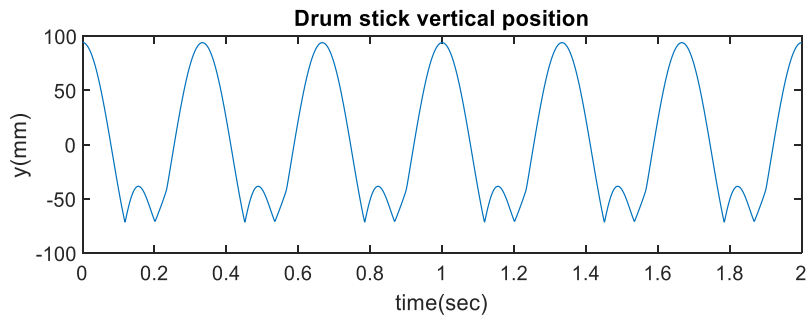

Fig. 5. Simulation:Vertical position of the drumstick in one trial after second learning phase $(\mathrm{f}=3 \mathrm{~Hz})$.

A comparison between simulation results and real data collected from a human drummer can show how the learning algorithm is similar to human behavior. In Figure 6, vertical position of the drumstick is illustrated during double stroke drum roll performed by a drummer. This data is collected from a drummer performing the double stroke task with both hands. However, the data from one hand can be compared to the simulations.

The double stroke task is learned for different frequencies. In Figure 7, the results for $f=6 \mathrm{~Hz}$ is shown. This interactive learning algorithm can refine the performance of the robot in real time, and the robot can easily adapt to the uncertainty and nonlinearity of the environment which is the drum kit in this application.

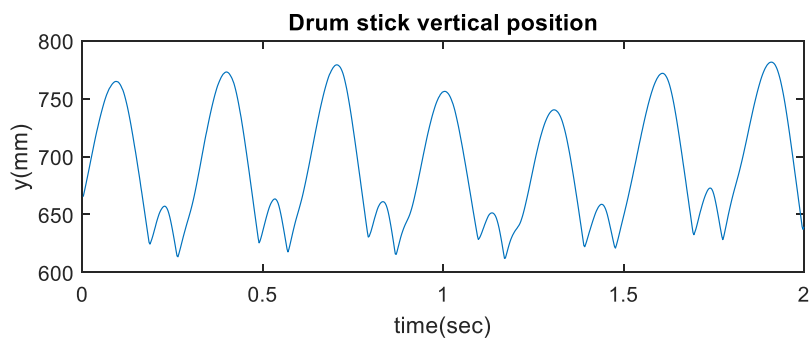

Fig. 6. Real Data: Vertical position of the drumstick during double stroke drum roll performed by a human drummer (raght hand).

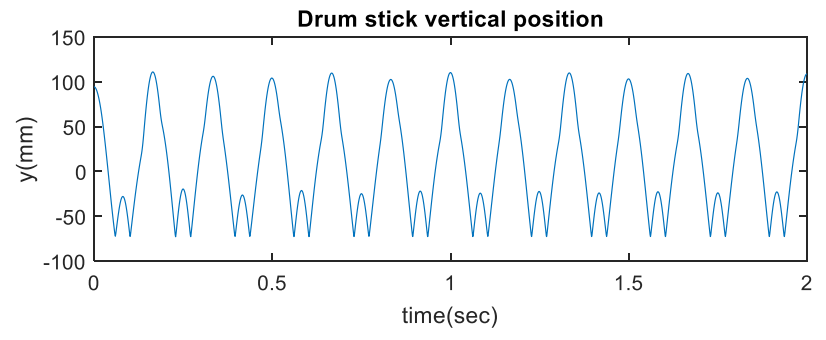

Fig. 7. Simulation: Vertical position of the drumstick for $f=6 \mathrm{~Hz}$.

\section{DISCUSSION AND CONCLUSION}

The results show that robots can learn to play drum by interaction with the environment, just like humans. The first point is that the interactive learning strategy does not need the analytical approach to perform well. We showed that with a learning method we can have remarkable performing results. The 2-DoF robot arm used in this study has a nonlinear dynamical behavior, which makes it very hard for analytical solutions. The only way to handle this nonlinearity is to use numerical methods. The learning technique is more robust and makes it possible to be used in real-time settings too.

Also, the uncertainties in the drum model and the vibrations of the drum membrane during drum roll inserts noise to the system. In our study the noise considered in the drum physical model did not affect the performance of the robot noticeably. Also, we have shown that the algorithm can adapt to drum kits with different physical attributes easily.

On the other hand, the simulation results are significantly compatible with the results reported by Hajian et al. They used a dataset collected from human drummers to show the relation between grip force and frequency. The same pattern can be seen in the trained data from simulations. It shows that the learning algorithm finds a natural solution to the problem which is close to the characteristics of human drummers.

Our comparison between simulation results and real data collected from a human drummer indicates considerable similarity (Figure 5 and 6). The human drummer exploits natural dynamics of the drumstick and drum membrane to perform the double stroke task. We can see that the second stroke in each period is hit completely passively. It means that the drummer only controls the joint stiffness to use the rebounding effect of the first stroke. The learning model which we introduced here, outputs behavior similar to the drummer behavior under the physical and control constraints imposed by the dynamical system.

In this study, we introduced a general approach to the problem of interactive learning for drumming by defining a basic task. The main advantage of this method is the use of the sound-based features for adjusting control parameters. For more accurate performance, there should be more components in the dynamical model, including more parameters that need to be learned. Alongside stiffness, the movement amplitude is a key factor in high speed drumming. We kept the amplitude fixed for all frequencies in this study because we wanted to keep it simple. However, in implementation, the amplitude adjustment should be considered.

In future work, the model will be implemented on a robotic arm prototype. The interactive learning scheme enables the robot to refine its performance in real-time and also makes it robust to the uncertainty of the environment. There is the 
possibility to try different drum kits for the same robot as long as the robot tries to learn interactively.

\section{ACKNOWLEDGMENT}

This work is supported by the Research Council of Norway through its Centres of Excellence scheme, project number: 262762.

\section{REFERENCES}

[1] N. Hogan, "Impedance control: An approach to manipulation: Part IIimplementation," Journal of Dynamic Systems, Measurement and Control, Transactions of the ASME, vol. 107, no. 1, pp. 8-16, Mar. 1985, doi: 10.1115/1.3140713.

[2] J. Buchli, F. Stulp, E. Theodorou, and S. Schaal, "Learning variable impedance control," The International Journal of Robotics Research, vol. 30, no. 7, pp. 820-833, Jun. 2011, doi: $10.1177 / 0278364911402527$.

[3] R. I. Godøy, M. Song, and S. Dahl, "Exploring Sound-Motion Textures in drum set performance," in Proceedings of the 14th Sound and Music Computing Conference, 2017.

[4] A. Karniel, "The minimum transition hypothesis for intermittent hierarchical motor control," Frontiers in Computational Neuroscience, vol. 7, no. FEB, Feb. 2013, doi: 10.3389/fncom.2013.00012.
[5] I. D. Loram, C. van de Kamp, M. Lakie, H. Gollee, and P. J. Gawthrop, "Does the Motor System Need Intermittent Control?," Exercise and Sport Sciences Reviews, vol. 42, no. 3, pp. 117-125, Jul. 2014, doi: 10.1249/JES.0000000000000018.

[6] P. Gawthrop, I. Loram, M. Lakie, and H. Gollee, "Intermittent control: A computational theory of human control," Biological Cybernetics, vol. 104, no. 1-2, pp. 31-51, Feb. 2011, doi: 10.1007/s00422-0100416-4.

[7] A. Z. Hajian, D. S. Sanchez, and R. D. Howe, "Drum roll: Increasing bandwidth through passive impedance modulation," in Proceedings IEEE International Conference on Robotics and Automation, 1997, vol. 3, pp. 2294-2299, doi: 10.1109/robot.1997.619304.

[8] E. Berdahl, E. Berdahl, B. Verplank, J. O. Smith, and I. G. Niemeyer, "A Physically Intuitive Haptic Drumstick," in Porceedings International Computer Music Conference, 2007.

[9] Y. G. Kim, M. Garabini, J. Park, and A. Bicchi, "Drum stroke variation using Variable Stiffness Actuators," in IEEE International Conference on Intelligent Robots and Systems, 2014, pp. 3892-3897, doi: 10.1109/IROS.2014.6943109.

[10] D. Gopinath and G. Weinberg, "A generative physical model approach for enhancing the stroke palette for robotic drummers," Robotics and Autonomous Systems, 2016, doi: 10.1016/j.robot.2016.08.020.

[11] R. I. Godøy, "Motor constraints shaping musical experence," Music Theory Online, vol. 24, no. 3. Society for Music Theory, 01-Sep-2018, doi: $10.30535 / \mathrm{mto} .24 .3 .8$. 